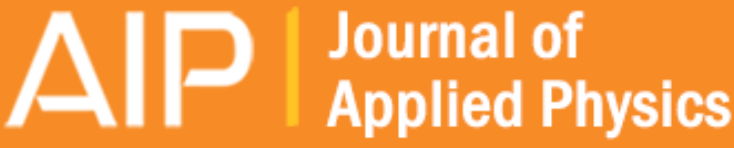

\section{Pressure induced phase transitions in TiH2}

Guoying Gao, Aitor Bergara, Guangtao Liu, and Yanming Ma

Citation: Journal of Applied Physics 113, 103512 (2013); doi: 10.1063/1.4795164

View online: http://dx.doi.org/10.1063/1.4795164

View Table of Contents: http://scitation.aip.org/content/aip/journal/jap/113/10?ver=pdfcov

Published by the AIP Publishing

\section{Articles you may be interested in}

High-pressure single-crystal elasticity study of $\mathrm{CO} 2$ across phase I-III transition

Appl. Phys. Lett. 104, 141901 (2014); 10.1063/1.4870526

X-ray diffraction study on pressure-induced phase transformations and the equation of state of ZnGa2Te4

J. Appl. Phys. 114, 233507 (2013); 10.1063/1.4851735

Novel pressure-induced phase transitions in $\mathrm{C} 03 \mathrm{O} 4$

Appl. Phys. Lett. 102, 041912 (2013); 10.1063/1.4790387

High pressure phase transition study of $\mathrm{B}-\mathrm{C}-\mathrm{N}$ compound

J. Appl. Phys. 107, 073508 (2010); 10.1063/1.3369279

Pressure- and temperature-induced phase transition in the $\mathrm{B}-\mathrm{C}$ system

J. Appl. Phys. 100, 013516 (2006); 10.1063/1.2209170

\section{Instruments for Advanced Science}

Contact Hiden Analytical for further details: w www.HidenAnalytical.com E info@hiden.co.uk CLICK TO VIEW our product catalogue

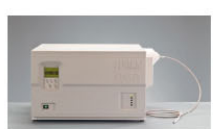

Gas Analysis

dynamic measurement of reaction gas streams catalysis and thermal analysis molecular beam studies

issolved species probes

fermentation, environmental and ecological studies

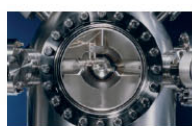

Surface Science

UHVTPD

SIMS end point detection in ion beam etch

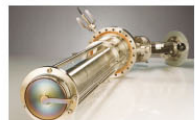

Plasma Diagnostics - plasma source characterization etch and deposition process reaction kinetic studies analysis of neutral and radical species

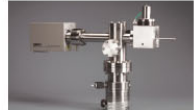

Vacuum Analysis partial pressure measurement and control of process gases reactive sputter process contro , vacuum diagnostics , Jacum coating process monitorin 


\title{
Pressure induced phase transitions in $\mathrm{TiH}_{2}$
}

\author{
Guoying Gao, ${ }^{1}$ Aitor Bergara, ${ }^{2,3,4}$ Guangtao Liu, ${ }^{1}$ and Yanming Ma ${ }^{1, a)}$ \\ ${ }^{1}$ State key Lab of Superhard Materials, Jilin University, Changchun 130012, People's Republic of China \\ ${ }^{2}$ Materia Kondentsatuaren Fisika Saila, Zientzia eta Teknologia Fakultatea, Euskal Herriko Unibertsitatea, \\ 644 Postakutxatila, 48080 Bilbo, Basque Country, Spain \\ ${ }^{3}$ Donostia International Physics Center (DIPC), Paseo de Manuel Lardizabal, 20018, Donostia, \\ Basque Country, Spain \\ ${ }^{4}$ Centro de Fisica de Materiales CSIC-UPV/EHU, 1072 Posta kutxatila, E-20080 Donostia, \\ Basque Country, Spain
}

(Received 11 September 2012; accepted 26 February 2013; published online 13 March 2013)

\begin{abstract}
Recent room temperature experiments on $\mathrm{TiH}_{2}$ [Kalita et al., J. Appl. Phys. 108, 043511 (2010)], an important compound in hydrogen storage research, revealed a cubic (fcc, $F m-3 m$ ) to tetragonal (bct, $I 4 / \mathrm{mmm}$ ) phase transition at around $0.6 \mathrm{GPa}$, which was suggested to remain stable up to at least $90 \mathrm{GPa}$. However, the simulated $X$-ray diffraction (XRD) pattern of the $I 4 / \mathrm{mmm}$ structure cannot explain all the diffraction peaks observed at $90 \mathrm{GPa}$. In this article, we apply the recently developed particle swarm optimization algorithm for crystal structure prediction to propose that at $63 \mathrm{GPa} \mathrm{TiH}{ }_{2}$ presents a further phase transition from $I 4 / \mathrm{mmm}$ to $P 4 / \mathrm{nmm}$, which we have also confirmed is dynamically and enthalpically stable up to $294 \mathrm{GPa}$. Moreover, the XRD patterns and calculated lattice parameters of the $P 4 / \mathrm{nmm}$ structure are in good agreement with the experimental available data. Above $294 \mathrm{GPa}$, we predict a monoclinic $P 2_{1} / m$ structure to be stable. (C) 2013 American Institute of Physics. [http://dx.doi.org/10.1063/1.4795164]
\end{abstract}

\section{INTRODUCTION}

High-pressure phases of many hydrides have been the subject of study for several years, ${ }^{1-11}$ which has been fundamental to identify new equilibrium and metastable structures, those could even be quenchable. Interestingly, metallic phases of some compressed hydrides have been predicted to be high temperature superconductors, ${ }^{2-8}$ and many others are promising compounds for hydrogen storage. ${ }^{9-11}$

In this article, we will characterize the structural phase sequence of $\mathrm{TiH}_{2}$ under pressure, which although itself is not the ideal candidate for hydrogen storage, it has been shown that it can catalyze and enhance the reversible hydrogenation and dehydrogenation processes required in hydrogen storage research. ${ }^{12-16}$ As it is common for many transition-metal dihydrides $\left(\mathrm{MH}_{2}\right)$, at room temperature $\mathrm{TiH}_{2}$ stabilizes in the $\mathrm{CaF}_{2}$-type (fcc) structure. However, below $17^{\circ} \mathrm{C}$ the fcc phase becomes unstable and transforms to a tetragonal $14 / \mathrm{mmm}$ structure. ${ }^{17}$ Similar instabilities have been also observed in other group IVB hydrides (e.g., $\mathrm{ZrH}_{2}$ and $\left.\mathrm{HfH}_{2}\right) .{ }^{18-20}$ Recent synchrotron X-ray diffraction (XRD) experiments of $\mathrm{TiH}_{2}$ at room temperature and high pressure revealed a phase transition from fcc to $14 / \mathrm{mmm}$ at $0.6 \mathrm{GPa}$, which was concluded to remain stable up to $90 \mathrm{GPa}$, as there was not identified any other structural transition in this pressure range. ${ }^{21}$ However, we have seen that the simulated XRD pattern of the $I 4 / \mathrm{mmm}$ structure cannot explain all the experimental diffraction peaks at $90 \mathrm{GPa}$. In this article, we have used our newly developed particle swarm optimization method for crystal structure prediction to extensively explore the high-pressure structures of $\mathrm{TiH}_{2}$ at zero temperature. Interestingly, we predict another

\footnotetext{
${ }^{\text {a) }}$ Author to whom correspondence should be addressed. Electronic mail: mym@jlu.edu.cn.
}

phase transition from $I 4 / \mathrm{mmm}$ to $P 4 / \mathrm{nmm}$ at $63 \mathrm{GPa}$, which fits better to the experimental XRD pattern and the resulting unit cell parameters. ${ }^{21}$

\section{COMPUTATIONAL DETAIL}

The crystal structure prediction is based on a global minimization of enthalpy surfaces merging ab initio totalenergy calculations as implemented in the Crystal structure AnaLYsis by Particle Swarm Optimization (CALYPSO) code, ${ }^{22}$ which has been successfully applied to predict several other structures at high pressure. ${ }^{23-25}$ The underlying $a b$ initio structural relaxations were performed using density functional theory within the Perdew-Burke-Ernzerh (PBE) of parameterization of the generalized gradient approximation $(\mathrm{GGA})^{26}$ as implemented in the Vienna ab initio simulation package $(\mathrm{VASP})^{27}$ code. The all-electron projector-augmented wave (PAW) ${ }^{28}$ method was adopted with $3 d^{2} 4 s^{2}$ and $1 s^{1}$ as valence electrons for $\mathrm{Ti}$ and $\mathrm{H}$ atoms, respectively. The energy cutoff $600 \mathrm{eV}$ and appropriate Monkhorst-Pack ${ }^{29}$ $k$ meshes were chosen to ensure that enthalpy calculations are well converged to better than $1 \mathrm{meV} /$ formula unit (f.u.). The phonon calculations were carried out using a supercell approach $^{30}$ with the PHONOPY code. ${ }^{31}$

\section{RESULTS AND DISCUSSION}

Structural predictions for $\mathrm{TiH}_{2}$ have then been performed with CALYPSO $^{22}$ using simulation sizes ranging from one to four formula units per primitive cell at $1 \mathrm{~atm}, 50,80,150$, $200,250,300,400$, and $450 \mathrm{GPa}$. We have also examined the structure searching convergence with variation of the simulation cell sizes. According to our simulations, at $1 \mathrm{~atm}$ and $50 \mathrm{GPa}, \mathrm{TiH}_{2}$ adopts a centered tetragonal structure with space group I4/mmm (2 f.u./cell) (Fig. 1(a)), which is in good 


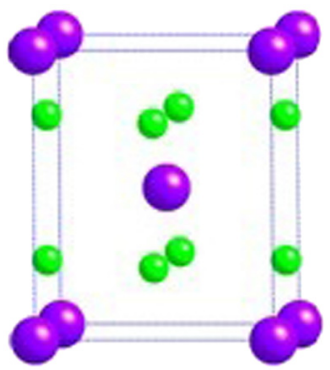

(a) $\mathrm{I} 4 / \mathrm{mmm}$

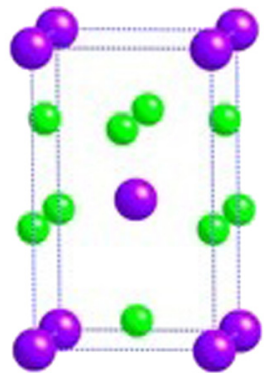

(b) $\mathrm{P} 4 / \mathrm{nmm}$

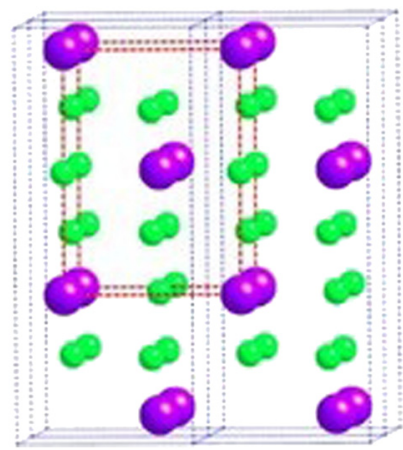

(c) $\mathbf{P} 2_{1} / \mathbf{m}$

FIG. 1. (a), (b), and (c) are the predicted $I 4 / \mathrm{mmm}, P 4 / \mathrm{nmm}$, and $P 2_{1} / \mathrm{m}$ structures for $\mathrm{TiH}_{2}$. Titanium and hydrogen atoms are shown with large and small balls, respectively. The $P 4 / \mathrm{nmm}$ structure contains 2 formula units for unit cell and the lattice parameters at $100 \mathrm{GPa}$ are $a=2.6033 \AA, c=4.3699 \AA$ with atomic positions of Ti at $2 c(0.5,0,0.26608)$, and $\mathrm{H}$ at $2 a(0,0,0)$ and $2 c(0,0.5,0.33139)$ sites.

agreement with the recent experiments. ${ }^{21}$ In this structure, the Ti atoms form a body-centered tetragonal (bct) sublattice and $\mathrm{H}$ atoms are located on the planes, forming a one dimensional chain. At the same time, we also predict a metastable fcc structure (i.e., a $\mathrm{CaF}_{2}$-type structure), which is the stable structure of many transition-metal dihydrides at ambient conditions. The most stable structure we have found at 80,150 , 200 , and $250 \mathrm{GPa}$, is a simple tetragonal $\mathrm{P} 4 / \mathrm{nmm}$ structure (Fig. 1(b)). In order to make it easier to compare with the $I 4 / \mathrm{mmm}$ structure, in Fig. 1(b) the $P 4 / \mathrm{nmm}$ structure is also presented with a bct cell. Besides the positions of the $\mathrm{H}$ atoms,

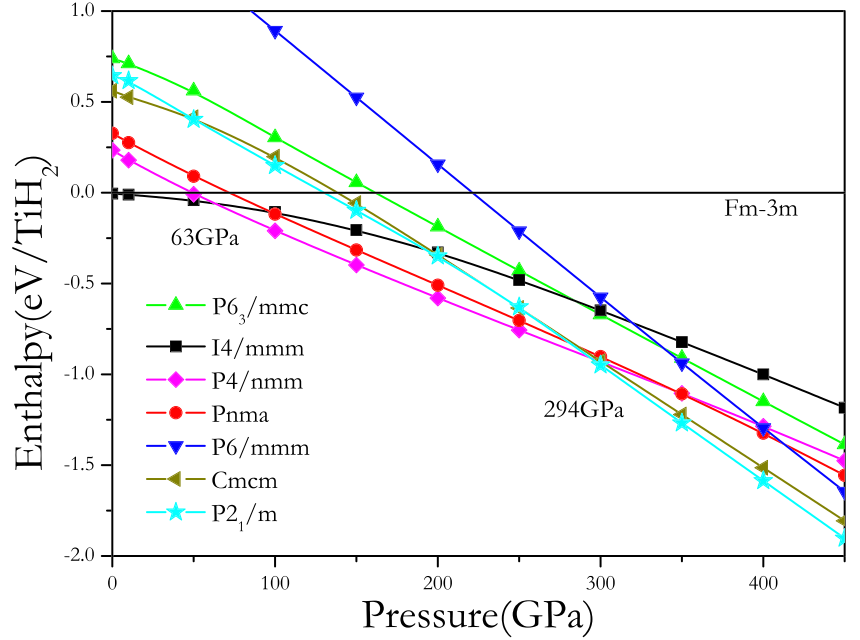

FIG. 2. The enthalpies per formula unit of various structures as a function of pressure with respect to the $F m-3 m$ structure.

an important difference between $I 4 / \mathrm{mmm}$ and $P 4 / \mathrm{nmm}$ structures is that in the latter one inner Ti atom is slightly deviated from the center of the cell. Considering that the diffraction cross-section of $\mathrm{H}$ atoms is extremely small, the XRD patterns of the $14 / \mathrm{mmm}$ and $P 4 / \mathrm{nmm}$ structures are expected to be very similar and, therefore, difficult to differentiate. At and above $300 \mathrm{GPa}$, we predict a monoclinic $P 2_{1} / m$ structure (4 f.u./cell) (Fig. 1(c)), which can also be seen as a little distorted bcc Ti lattice.

To check the enthalpical stability of the resulting structures, Fig. 2 presents their enthalpy difference with respect to the fcc structure as a function of pressure. Due to the high pressure structures are very similar to each other and all the $\mathrm{H}$ atoms exist in the atomic form, we do not expect including the zero point energy will substantially change the calculated phase transition sequence. ${ }^{34}$ At zero pressure, the $I 4 / \mathrm{mmm}$ structure is just $5 \mathrm{meV}$ lower in enthalpy than that of the fcc phase. Actually, at low temperature, $\mathrm{TiH}_{2}$ stabilizes in the I4/mmm structure, but it becomes unstable at higher temperature, transforming from $I 4 / \mathrm{mmm}$

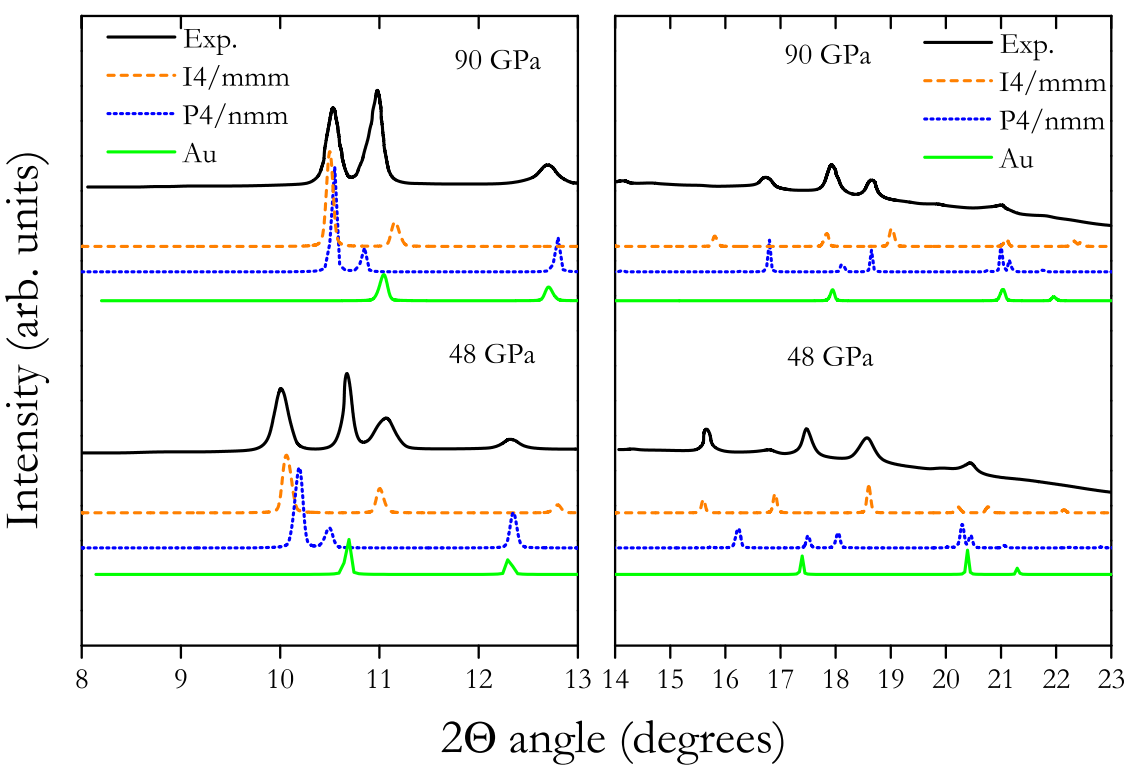

FIG. 3. The simulated XRD patterns of Au, I4/ $\mathrm{mmm}$, and $P 4 / \mathrm{nmm}-\mathrm{TiH}_{2}$ at 48 and $90 \mathrm{GPa}$ compared with the experimental data from Ref. 21, where we have considered the same $\mathrm{x}$-ray wavelength $(0.413561 \AA)$ as in the experiment. 


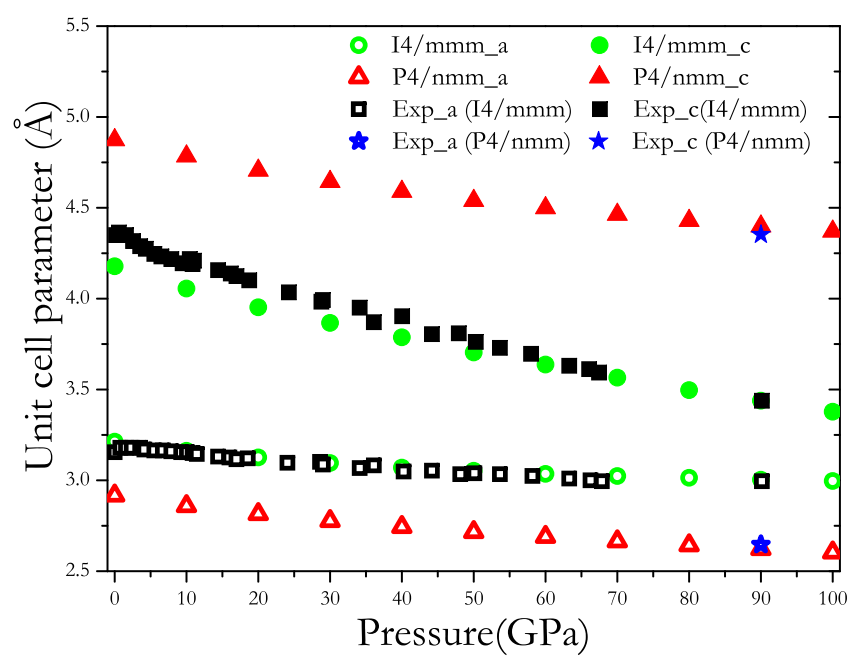

FIG. 4. Pressure evolution of the calculated and experimental unit cell parameters of the tetragonal $I 4 / \mathrm{mmm}$ and $P 4 / \mathrm{nmm}$ phases of $\mathrm{TiH}_{2}$. Solid and hollow represent parameters $c$ and $a$, respectively. Green circles and red triangles refer to the calculated data with $I 4 / \mathrm{mmm}$ and $P 4 / \mathrm{nmm}$, respectively. Black squares are the parameters obtained fitting the experimental XRD data considering the Miller indices for the $14 / \mathrm{mmm}$ structure (Ref. 21), while blue stars are the fitted parameters when the Miller indices for the $P 4 / \mathrm{nmm}$ structure are considered.

to fcc at $17^{\circ} \mathrm{C}$. According to our calculations, at zero temperature, the $I 4 / \mathrm{mmm}$ structure is enthalpically stable up to about $63 \mathrm{GPa}$, above which it transforms to another tetragonal $P 4 / \mathrm{nmm}$ phase. The $P 4 / \mathrm{nmm}$ structure has a very large stability range, from 63 to $294 \mathrm{GPa}$, and at higher pressures it transforms to a monoclinic $P 2_{1} / m$ structure. We have also considered the Pnma and P6/mmm structures, the highpressure structures of heavy alkali earth dihydrides, such as $\mathrm{CaH}_{2}{ }^{32}$ and $\mathrm{BaH}_{2},{ }^{33}$ but they are unstable with respect to our predicted $P 2_{1} / m$. Our predicted phase transition from $I 4 / \mathrm{mmm}$ to $P 4 / \mathrm{nmm}$ at $63 \mathrm{GPa}$ apparently contradicts the experimental results ${ }^{21}$ which claim that the $I 4 / \mathrm{mmm}$ structure persists up to at least $90 \mathrm{GPa}$. To further verify the validity of our predicted $P 4 / \mathrm{nmm}$ structure above $63 \mathrm{GPa}$, in Fig. 3 we compare the simulated XRD spectra of the $P 4 / \mathrm{nmm}$ and $I 4 / \mathrm{mmm}$ structures with the experimental data. Note that we used the break in Fig. 3 due to that the experimental work did not present any data between $13^{\circ}$ and $14^{\circ}$ and, in fact, there are no any peaks during this angle range in our simulations. At $48 \mathrm{GPa}$, the XRD data of $14 / \mathrm{mmm}$ $\mathrm{TiH}_{2}$ and $\mathrm{Au}$, which is used as a pressure marker in the experiment, perfectly reproduce the observed one, which supports the stability of the $I 4 / \mathrm{mmm}$ structure at this pressure, in accordance with our computed enthalpy curves. However, at $90 \mathrm{GPa}$, the XRD patterns of I4/ $m m m-\mathrm{TiH}_{2}$ and $\mathrm{Au}$ cannot explain all the experimental peaks, especially those located between $16^{\circ}$ and $17^{\circ}$. Interestingly, our predicted $P 4 / \mathrm{nmm}$ structure, which according to our calculations is enthalpically stable between 63 and $294 \mathrm{GPa}$, reproduces all the experimental diffraction peaks, so that we conclude it can be attributed to the $P 4 / \mathrm{nmm}$ structure and not to the low-pressure I4/ mmm phase.

We have also compared our calculated unit cell parameters with those that best fit the experimental XRD pattern. As it is shown in Fig. 4, below 63 GPa the computed unit cell parameters for $14 / \mathrm{mmm}$ are in good agreement with that obtained from the experimental XRD data, while the calculated unit cell parameters for $P 4 / \mathrm{nmm}$ at $90 \mathrm{GPa}$ have a very large difference from those obtained from the experimental XRD data when the fitting is done with the $I 4 / \mathrm{mmm}$ structure. But we should remember that it is not reasonable to fit the experimental XRD pattern with Miller indices corresponding to the low-pressure $14 / \mathrm{mmm}$ structure after $\mathrm{TiH}_{2}$ has transformed to $P 4 / \mathrm{nmm}$ above $63 \mathrm{GPa}$. Therefore, when the fitting to the experimental XRD pattern is done with Miller indices corresponding to the $P 4 / \mathrm{nmm}$ structure, the agreement with the calculated unit cell parameters is excellent, as the blue stars in Fig. 4 show.

Finally, we need to check the dynamical stability of the proposed $P 4 / \mathrm{nmm}$ structure. Fig. 5 shows the phonon dispersion curves of the $P 4 / \mathrm{nmm}$ structure at 50 and $300 \mathrm{GPa}$, respectively. The absence of imaginary frequency suggests that $P 4 / \mathrm{nmm}$ is dynamically stable within its enthalpically stability range, which further supports the validity of this structure.
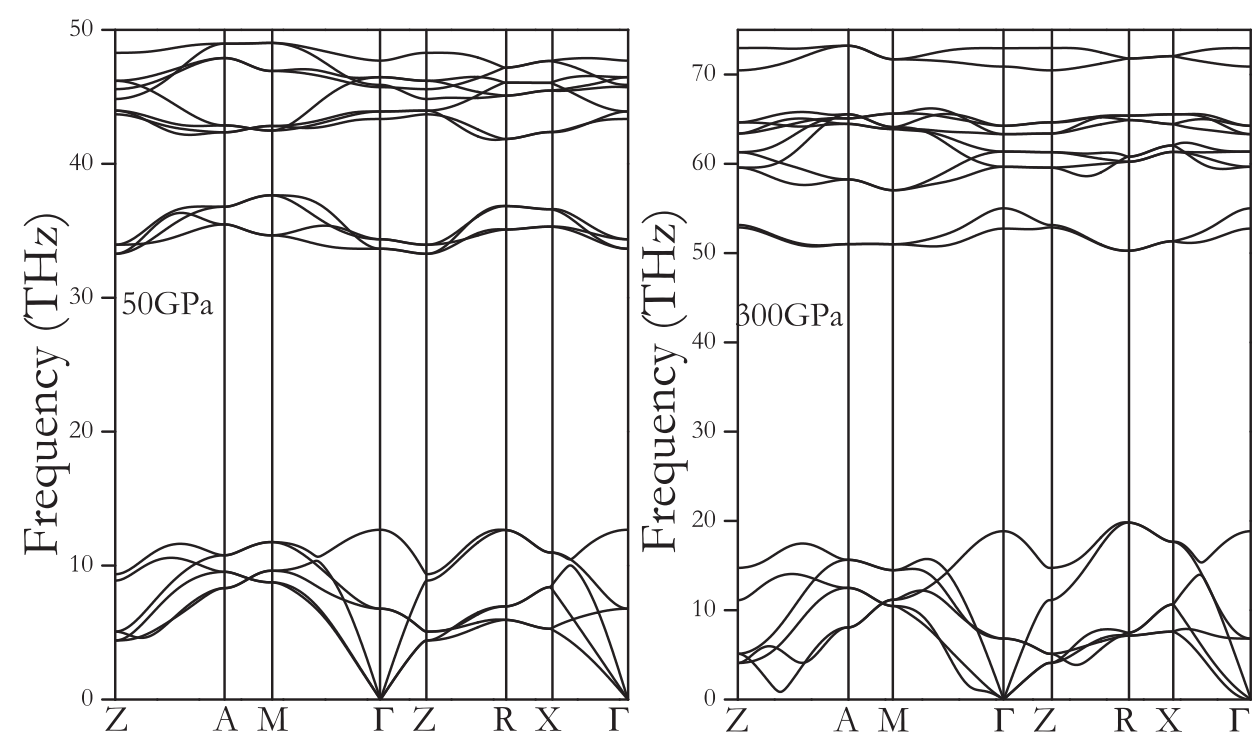

FIG. 5. Phonon dispersion curves for the $\mathrm{P} 4 / \mathrm{nmm}$ structure at 50 and $300 \mathrm{GPa}$. 


\section{CONCLUSION}

In conclusion, zero temperature ab initio enthalpy calculations based on the structures predicted by CALYPSO suggest that $\mathrm{TiH}_{2}$ adopts a body centered tetragonal $I 4 / \mathrm{mmm}$ structure at ambient pressure and transforms to a simple tetragonal $P 4 / \mathrm{nmm}$ phase at $63 \mathrm{GPa}$, which remains stable up to 294 GPa when $P 2_{1} / m$ structure becomes preferred. The comparison between the experimental and calculated X-ray diffraction patterns and the fitted and calculated unit cell parameters allow us to confirm the phase transition from I4/ $\mathrm{mmm}$ to $P 4 / \mathrm{nmm}$ below $90 \mathrm{GPa}$.

\section{ACKNOWLEDGMENTS}

We acknowledge funding supports from the National Natural Science Foundation of China (under Grant Nos. 91022029 and 11025418), the research fund of Key Laboratory of Surface Physics and Chemistry (No. SPC201103), and the China 973 Program under Grant No. 2011CB808200. A.B. acknowledges the Department of Education, Universities and Research of the Basque Government, UPV/EHU (Grant No. IT-366-07) and the Spanish Ministry of Science and Innovation (Grant No. FIS2010-19609-C02-00).

${ }^{1}$ O. Degtyareva, M. Martinez-Canales, A. Bergara, X. J. Chen, Y. Song, V. V. Struzhkin, H. K. Mao, and R. J. Hemley, Phys. Rev. B 76, 064123 (2007).

${ }^{2}$ G. Gao, A. R. Oganov, A. Bergara, M. Martinez-Canales, T. Cui, T. Iitaka, Y. Ma, and G. Zou, Phys. Rev. Lett. 101, 107002 (2008).

${ }^{3}$ G. Gao, A. R. Oganov, P. Li, Z. Li, H. Wang, T. Cui, Y. Ma, A. Bergara, A. O. Lyakhov, T. Iitaka, and G. Zou, Proc. Natl. Acad. Sci. U.S.A. 107, 1317 (2010).

${ }^{4}$ G. Gao, H. Wang, A. Bergara, Y. Li, G. Liu, and Y. Ma, Phys. Rev. B 84, 064118 (2011).

${ }^{5}$ M. Martinez-Canales, A. R. Oganov, Y. Ma, Y. Yan, A. O. Lyakhov, and A. Bergara, Phys. Rev. Lett. 102, 087005 (2009).

${ }^{6}$ J. S. Tse, Y. Yao, and K. Tanaka, Phys. Rev. Lett. 98, 117004 (2007).
${ }^{7}$ X. J. Chen, J. L. Wang, V. V. Struzhkin, H. Mao, R. J. Hemley, and H. Lin, Phys. Rev. Lett. 101, 077002 (2008).

${ }^{8}$ D. Y. Kim, R. H. Scheicher, C. J. Pickard, R. J. Needs, and R. Ahuja, Phys. Rev. Lett. 107, 117002 (2011)

${ }^{9}$ B. Sakintuna, F. Lamari-Darkrim, and M. Hirscher, Int. J. Hydrogen Energy 32, 1121 (2007).

${ }^{10}$ B. Li, Y. Ding, D. Y. Kim, R. Ahuja, G. Zou, and H. Mao, Proc. Natl. Acad. Sci. U.S.A. 108, 18618 (2011).

${ }^{11}$ L. Schlapbach and A. Zuttel, Nature 414, 353 (2001).

${ }^{12}$ Y. Fukai, Metal-Hydrogen System (Springer-Verlag, Berlin, 2005).

${ }^{13}$ T. Yildirim and S. Ciraci, Phys. Rev. Lett. 94, 175501 (2005).

${ }^{14}$ P. Wang, X.-D. Kang, and H.-M. Cheng, J. Phys. Chem. B 109, 20131 (2005).

${ }^{15}$ J. Iñiguez, T. Yildirim, T. J. Udovic, M. Sulic, and C. M. Jensen, Phys. Rev. B 70, 060101 (2004)

${ }^{16}$ G. Sandrock, K. Gross, and G. Thomas, J. Alloys Compd. 339, 299 (2002).

${ }^{17}$ P. E. Kalita, A. L. Cornelius, K. E. Lipinska-Kalita, C. L. Gobin, and H. Peter Liermann, J. Phys. Chem. Solids 69, 2240 (2008).

${ }^{18}$ K. Miwa and A. Fukumoto, Phys. Rev. B 65, 155114 (2002).

${ }^{19}$ R. Quijano, R. de Coss, and D. J. Singh, Phys. Rev. B 80, 184103 (2009).

${ }^{20}$ Q. Xu and A. Vander Ven, Phys. Rev. B 76, 064207 (2007).

${ }^{21}$ P. E. Kalita, S. V. Sinogeikin, K. Lipinska-Kalita, T. Hartmann, X. Z. Ke, C. F. Chen, and A. Cornelius, J. Appl. Phys. 108, 043511 (2010).

${ }^{22}$ Y. Wang, J. Lv, L. Zhu, and Y. Ma, Phys. Rev. B 82, 094116 (2010).

${ }^{23}$ P. Li, G. Gao, Y. Wang, and Y. Ma, J. Phys. Chem. C 114, 21745 (2010).

${ }^{24}$ J. Lv, Y. Wang, L. Zhu, and Y. Ma, Phys. Rev. Lett. 106, 015503 (2011).

${ }^{25}$ L. Zhu, H. Wang, Y. Wang, J. Lv, Y. Ma, Q. Cui, Y. Ma, and G. Zou, Phys. Rev. Lett. 106, 145501 (2011).

${ }^{26}$ J. P. Perdew, K. Burke, and M. Ernzerhof, Phys. Rev. Lett. 77, 3865 (1996).

${ }^{27}$ G. Kresse and J. Furthmüller, Phys. Rev. B 54, 11169 (1996).

${ }^{28}$ G. Kresse and D. Joubert, Phys. Rev. B 59, 1758 (1999).

${ }^{29}$ H. J. Monkhorst and J. D. Pack, Phys. Rev. B 13, 5188 (1976).

${ }^{30}$ K. Parlinski, Z. Q. Li, and Y. Kawazoe, Phys. Rev. Lett. 78, 4063 (1997).

${ }^{31}$ A. Togo, F. Oba, and I. Tanaka, Phys. Rev. B 78, 134106 (2008).

${ }^{32}$ Y. Li, B. Li, T. Cui, Y. Li, L. Zhang, Y. Ma, and G. Zou, J. Phys.: Condens. Matter 20, 045211 (2008).

${ }^{33}$ K. Kinoshita, M. Nishimura, Y. Akahama, and H. Kawamura, Solid State Commun. 141, 69 (2007).

${ }^{34}$ G. Gao, H. Wang, L. Zhu, and Y. Ma, J. Phys. Chem. C 116, 1995 (2012). 